\title{
APPLICATION DES EQUATIONS INTEGRALES D'HELMHOLTZ À L'OPTIMISATION D'UNE SONDE INTENSIMÉTRIQUE 3D SPHÉRIQUE
}

\author{
O. COSTE, J.C. PATRAT et R. HENRY* \\ Laboratoire d'Etudes Aérodynamiques (URA 191), C.EA.T, 43 route de l'Aérodrome, \\ F-86036 Poitiers, France \\ ${ }^{*}$ Bâtiment $H, 40$ avenue du Recteur Pineau, F-86022 Poitiers, France
}

\begin{abstract}
Various acoustic probes are designed to reduce scattering. On the contrary, the spherical probe allowing $3 D$ acoustic intensity measurements perturbs the acoustic field in a well-known and useful way. Its measurement principle assumes that the sphere is the only part which scatters the field. The aim of the present work is to quantify and to reduce the scattering of the handle.

résumé: les diverses sondes acoustiques sont conçues pour minimiser les effets de diffraction. Au contraire, la sonde sphérique permettant des mesures $d$ 'intensité acoustique en trois dimensions perturbe le champ acoustique de façon connue et exploitable. Son principe de mesure suppose que la sphère est la seule partie diffractante de la sonde. Le but de cette étude est de quantifier puis de minimiser les phénomènes de diffraction engendrés par la poignée.
\end{abstract}

\section{INTRODUCTION}

L'intérêt d'une sonde sphérique pour la détermination des trois composantes du vecteur intensité acoustique a été montré dans [1]. La connaissance analytique des phénomènes de diffraction autour de la sphère dans le cas d'un champ d'ondes planes ou d'ondes sphériques a permis de mettre au point des algorithmes conduisant à la connaissance complète du champ acoustique non perturbé. Comme pour l'intensimétrie traditionnelle par différences finies, ces derniers s'appuient sur des mesures de phases.

La direction de propagation ou encore les cosinus directeurs du vecteur intensité acoustique peuvent être déterminés jusqu'à $12500 \mathrm{~Hz}$.

A ces fréquences, les phénomènes de diffraction ne relèvent plus simplement de la sphère de mesure mais de toute la sonde. L'approche analytique n'étant plus possible pour cette géométrie complexe, la présente étude propose une approche numérique basée sur l'équation intégrale de Helmholtz.

Le but de cette étude est double:

- Dans un premier temps, ce calcul permet de quantifier les perturbations dues à la poignée et ainsi de connaître les limites de la géométrie présentée au $1{ }^{\text {er }}$ Congrès Français d'Acoustique [2].

- Dans un second temps, une optimisation de la forme de la poignée conduisant à une erreur minimale est proposée.

Même si ce calcul est limité à $10 \mathrm{kHz}$ pour les raisons présentées dans $\S \mathrm{I}$, ces enseignements restent valables dans tout le domaine de mesure.

\section{RESOLUTION PAR EQUATIONS INTEGRALES}

Les phénomènes de diffraction des ondes acoustiques peuvent être mis en équation sous forme intégrale par une combinaison de potentiels de simple et double couche. Soient $P_{d}$ la pression diffractée par l'obstacle et $P_{i}$ la pression incidente;

La fonction de Green s'écrit: $\quad G(M, P)=\frac{e^{i k\|\overrightarrow{M P}\|}}{4 \pi\|\overrightarrow{M P}\|}$ 
$\Sigma$ représente la surface de l'obstacle, $\Omega_{i}$ et $\Omega_{e}$ les domaines intérieur et extérieur.

$$
\int_{\Sigma}\left\{P_{\mathrm{d}}(\mathrm{P}) \frac{\partial \mathrm{G}(\mathrm{M}, \mathrm{P})}{\partial \mathrm{n}_{\mathrm{P}}}-\frac{\partial P_{\mathrm{d}}(\mathrm{P})}{\partial \mathrm{n}_{\mathrm{P}}} \mathrm{G}(\mathrm{M}, \mathrm{P})\right\} \mathrm{d} \Sigma=v P_{\mathrm{d}}(\mathrm{M})
$$

avec $v=0$ si $M \in \Omega_{i}, v=1$ si $M \in \Omega_{e}, v=\frac{1}{2}$ si $M \in \Sigma$.

L'étude étant réalisée dans l'hypothèse d'un champ incident d'ondes planes, le gradient de pression sur $\Sigma$ est donné par:

$$
\frac{\partial P_{d}(P)}{\partial n_{P}}=-\frac{\partial P_{1}(P)}{\partial n_{P}}
$$

L'intérêt principal [3] du polyèdre (approximation de la surface $\Sigma$ en éléments plans)

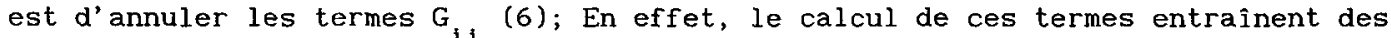
difficultés lors du découpáge de $\Sigma$ en éléments de surfaces gauches.

La pression ainsi que la composante normale de son gradient sont approximées par des distributions constantes par morceaux.

La pression diffractée en chacun des points $P$, centre de gravité des mailles est alors solution du sytème d'équations linéaires' suivant:

$$
\left(\frac{1}{2}[I]-[G]\right)\left[P_{d}\right]=[M]\left[\frac{\partial P_{1}}{\partial n}\right]
$$

avec pour $i \neq j$

$$
G_{1 j}=\int_{\Sigma_{j}}^{\frac{\partial G\left(P_{1}, P\right)}{\partial n_{j}}} d \Sigma_{j}
$$

pour $i=j$

$$
G_{1 i}=0
$$

$$
\begin{aligned}
& M_{11}=\int_{\Sigma_{j}} G\left(P_{1}, P\right) d \Sigma_{j} \\
& M_{11}=\lim _{\sigma_{i} \rightarrow 0} \int_{\Sigma_{1}-\sigma_{i}} G\left(P_{i}, P\right) d \Sigma_{1}
\end{aligned}
$$

Pour certains nombres d'ondes $k$ ', le noyau de l'équation (2) devient singulier. Au contraire de géométries simples, comme la sphère ou le cylindre, il n'est pas possible ici d'exprimer analytiquement ces fréquences. Schenck [4] a montré que ces valeurs $k^{\prime}$ sont celles pour lesquelles le problème intérieur de Dirichlet (7) admet une solution non triviale.

$$
\begin{cases}\Delta P_{d}(M)+k^{\prime 2} P_{d}(M)=0 & \text { pour } M \in \Omega_{i} \\ P_{d}(M)=0 & \text { pour } M \in \Sigma\end{cases}
$$

Pour remédier à ce problème, de la même façon que [3], des points à l'intérieur du solide sont utilisés pour surdéterminer le système. Cette technique bien adaptée pour les premiers nombres d'ondes caractéristiques, sera moins satisfaisante au delà de $10 \mathrm{kHz}$. En effet, la densité plus grande de fréquences caractéristiques entraînera une surdétermination plus importante et donc un conditionnement du système de plus en plus délicat.

En outre, le faible rayon de la tige (figure 1) donne à la sonde un comportement semblable à celui de deux obstacles côte à côte (sphère + poignée). L'apparition de divergences au voisinage des nombres d'ondes caractéristiques dépendra alors de 1 'incidence de I'onde.

\section{DIFFRACTION PAR LA SONDE 3D}

Le maillage de la sonde a été réalisé à l'aide de triangles, de trapèzes et de rectangles; L'utilisation de ces différentes formes a permis d'obtenir des mailles de surfaces comparables. La surface de la sphère est approximée par 55 triangles et 5 trapèzes assurant le raccordement avec la tige. Le maillage de la totalité de la sonde en 295 éléments (figure 1) est suffisant pour satisfaire les hypothèses du $§ I$. 


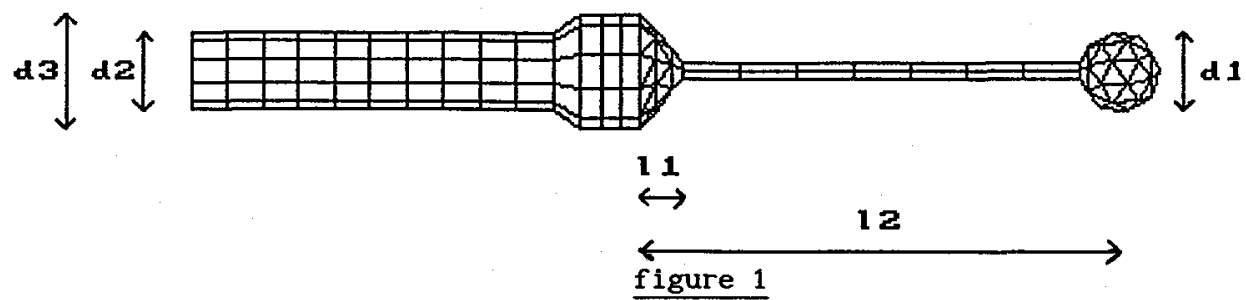

La méthode présentée dans § I permet de calculer la pression diffractée à la surface de la sonde ainsi que la pression mesurée par chacun de six microphones.

Soient $P$ et $P$, les pressions mesurées respectivement pour la sonde totale et pour la sphèrét seule. ${ }^{m s}$ De la même façon, $\Delta \varphi_{t}$ et $\Delta \varphi_{s}$ représentent le déphasage entre deux microphones d'un même axe de mesure.

Les perturbations engendrées par la poignée sont alors représentées par les grandeurs $\mathrm{K}$ et $\varphi$ :

$$
\mathrm{K}=20 \log \frac{\left|P_{\mathrm{mt}}\right|}{\left|P_{\mathrm{ms}}\right|} \quad \varphi=\Delta \varphi_{\mathrm{t}}-\Delta \varphi_{\mathrm{s}}
$$

L'évolution de $\varphi$ avec l'incidence de l'onde montre que les ondes provenant de l'avant dans l'axe de la tige (figure 2 ) sont beaucoup plus perturbées que celles provenant de l'arrière (figure 3 ). Le facteur $K$ évolue de façon similaire avec des variations maximums de $\pm 2.5 \mathrm{db}$.

Cette évolution en fonction de l'incidence de l'onde est vérifiée expérimentalement (figure 4 et 5). Deux raisons expliquent l'écart entre les deux courbes de la figure 5:

- de multiples réflexions non maîtrisées entre la sonde et le haut-parleur, - l'hypothèse de I'onde sphérique serait ici plus réaliste [5].

\section{I OPTIMISATION}

Les valeurs d2 et 12 (figure 1) ne peuvent pas pour des raisons technologiques être modifiées. En effet, la poignée contient les préamplificateurs et l'augmentation de 12 altèrerait la sensibilité de la chaine de mesure [2]. Le diamètre d1 de la sphère est quand à lui inversement proportionnel à la limite haute fréquence.

Ce calcul montre que l'influence du rayon de la tige est négligeable. Au contraire, la longueur du cône 11 et le diamètre de la fixation d3 jouent un rôle primordial. En prenant $\mathrm{d} 3=\mathrm{d} 2$ et en augmentant la longueur 11 , les phénomènes de diffraction engendrés par la poignée deviennent négligeables devant ceux engendrés par la sphère (figure 6 et 7 ).

Pour la géométrie optimisée (figure 8), la zone de fixation ou les surfaces fonctionnelles nécessaires à une orientation et à un positionnement précis sont placées à l'arrière de la sonde.

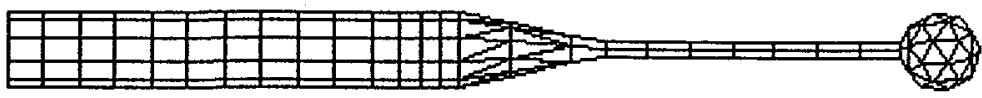

figure 8

\section{CONCLUSION}

L'utilisation des méthodes intégrales, pour une forme donnée de sonde intensimétrique, montre que les phénomènes de diffraction ne sont pas, en général, négligeables notamment en ce qui concerne les mesures de phases.

Une géométrie minimisant la diffraction de la poignée a ainsi pu être proposée. La sphère est alors la partie diffractante prépondérante ce qui justifie l'utilisation de l'algorithme présenté en [1]. 


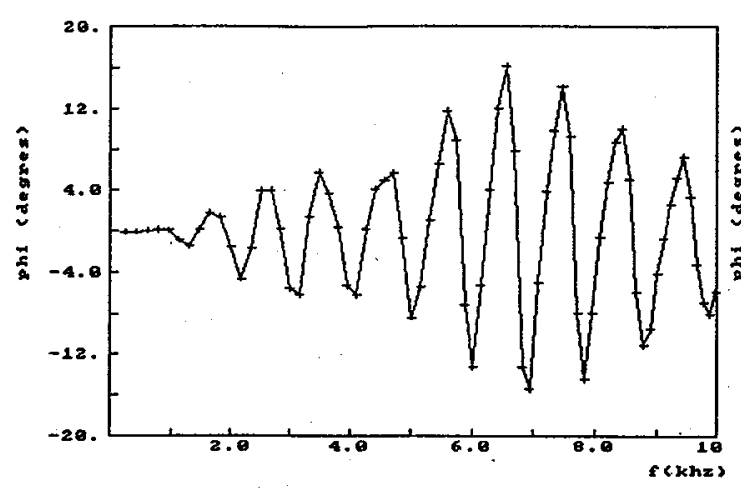

fig 2: forwards wave

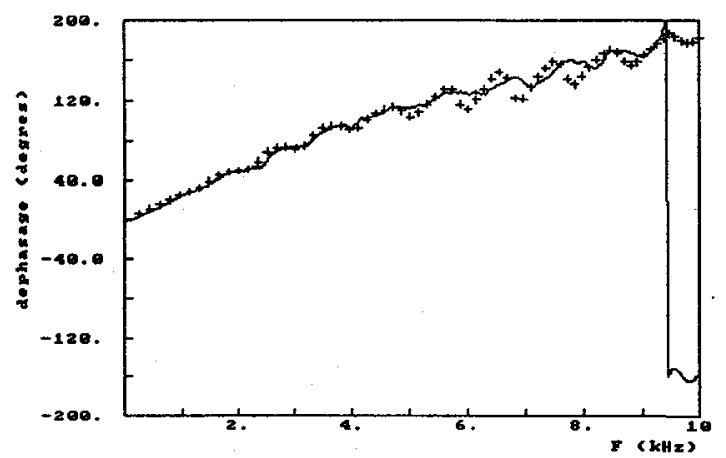

fig 4: forwards wave ++ theory, - experiment

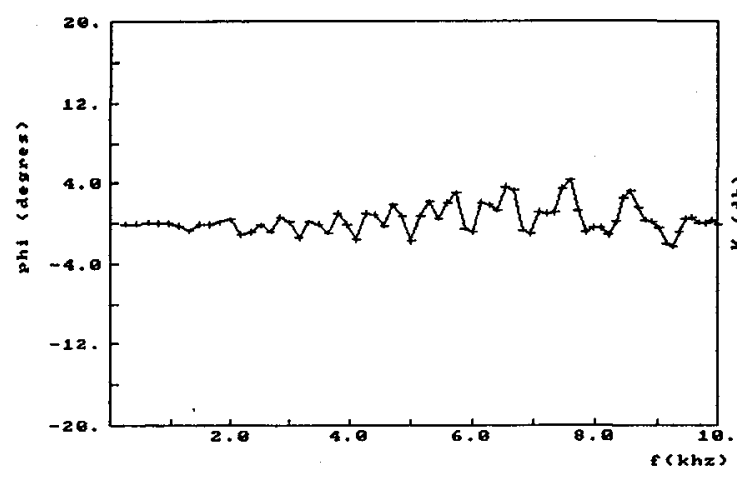

fig 6: forwards wave optimized probe

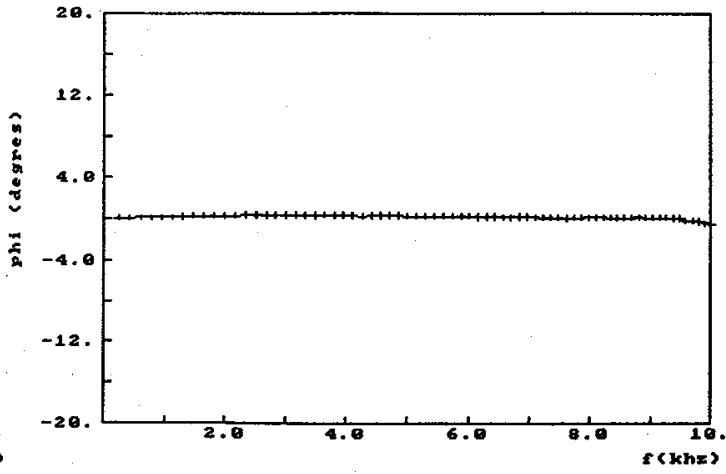

fig 3: backwards wave

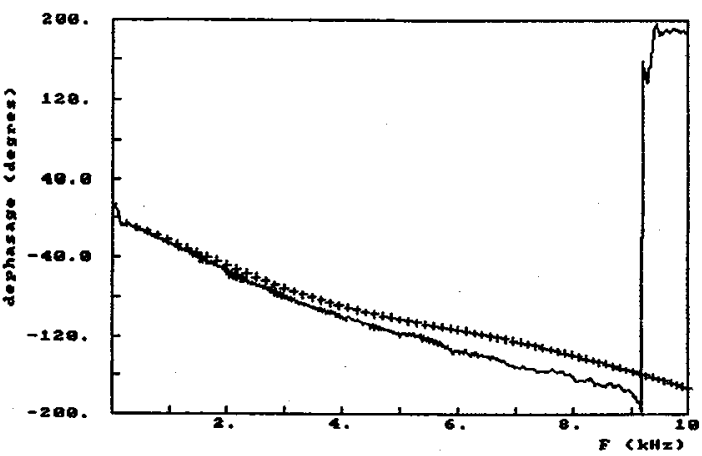

fig 5: backwards wave ++ theory, - experiment

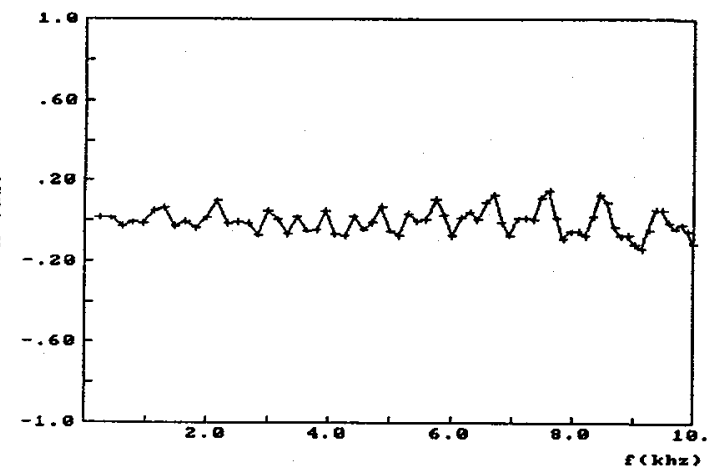

fig 7: forwards wave optimized probe

[1] O Coste, J.C Patrat "diffraction autour d'une sphére appliquée à une sonde intensimétrique 3D" $1^{\text {er }}$ Congrés Français d'Acoustique (895-898) Avril 1990.

[2] J Wasmer "sonde pour l'analyse d'intensimétrie à 3 dimensions" 1 er Congrés Français d'Acoustique (927-930) Avril 1990.

[3] G.H Koopman, H Brenner "method for computing the sound power of machines based on the Helmholtz integral" J.A.S.A (71) Janvier 1982.

[4] H.A Schenck "improved integral formulation for acoustic radiation problems" J.A.S.A (44) (41-58) décembre 1967.

[5] O Coste, J.C Patrat "scattering of a spherical wave applied to a $3 D$ sound intensity probe" Congrès INTERNOISE Sydney Décembre 1991. 